\title{
Ulcerative colitis six years after colon cancer: only a coincidence?
}

This article was published in the following Dove Press journal:

International Medical Case Reports Journal

29 April 2014

Number of times this article has been viewed

\author{
Minas Sakellakis' \\ Thomas Makatsoris' \\ Maria Gkermpesi \\ Stavros Peroukidis' \\ Haralabos Kalofonos' \\ 'Division of Oncology, Department of \\ Medicine, ${ }^{2}$ Department of Pathology, \\ University, Hospital of Patras, Patras, \\ Greece
}

\begin{abstract}
The association between inflammatory bowel disease and colorectal cancer is well known. Ulcerative colitis is a risk factor for the development of colorectal cancer, and this risk increases with the activity and duration of bowel inflammation. Here we describe the case of a 52-year-old man who developed ulcerative colitis 6 years after the diagnosis and treatment of colon cancer. Although this could be a coincidence, there could be additional possibilities, like pre-existence of quiescent colitis, late effect of therapy, or maybe the existence of common pathogenetic factors contributing to the development of ulcerative colitis and colorectal cancer.
\end{abstract}

Keywords: ulcerative, colitis, colorectal, cancer, inflammation

\section{Background}

Ulcerative colitis is an inflammatory bowel disease that affects 8-246 per 100,000 individuals with equal distribution between men and women. ${ }^{1}$ Although the exact cause is not fully understood, the predominant explanation is that there is an abnormal response of the bowel mucosa to stimuli caused by pathogens of the normal intestinal flora. ${ }^{2}$ The end result is an excessive and inappropriate sequence of immune events. ${ }^{2}$ Colorectal cancer (CRC) is the third leading cause of cancer-related deaths in the USA. ${ }^{3}$ The three most common conditions associated with CRC include the hereditary syndromes of familial adenomatous polyposis, hereditary nonpolyposis CRC, and inflammatory bowel disease. ${ }^{3,4}$ However, more than $95 \%$ of cases of $\mathrm{CRC}$ are seen in patients without significant risk factors. Potential risk factors for sporadic CRC include sedentary lifestyle, age, male sex, increased fat consumption, and obesity. ${ }^{4}$

It is well known that ulcerative colitis is associated with an increased risk of developing CRC. It is believed that the increased risk is due to longstanding inflammation of the intestine. ${ }^{5}$ Regarding ulcerative colitis, multiple risk factors have been proposed for the progression to CRC. It is widely known that the longest duration and extent of disease represent risk factors. Recent studies also suggest that the degree of inflammation is directly related to increased risk of dysplasia and development of CRC. ${ }^{6}$ Additionally, the concomitant presence of primary sclerosing cholangitis increases the risk of developing CRC. Although not always the case, progression to carcinogenesis usually follows a specific sequence of events, leading to a progressively greater number and degree of dysplastic lesions, and finally to carcinogenesis. ${ }^{5}$ It is also known however, that there is a wide range and variety of manifestations and natural history in inflammatory bowel disease-related CRC. ${ }^{7}$
Correspondence: Minas Sakellakis Division of Oncology, Department of Medicine, University Hospital of Patras, 26504, Rion-Patras, Greece

Tel +302610999535

Fax +30261099 4645

Email doctorsakellakis@gmail.com 
Here we present the case of a patient who developed symptoms of ulcerative colitis 6 years after he was diagnosed and treated for CRC.

\section{Case presentation}

A 52-year-old man with a history of hypertension and no known history of ulcerative colitis or other remarkable gastrointestinal complaints was admitted to the University Hospital of Patras due to hematochezia. He underwent a colonoscopy and a mass was found at the lower sigmoid colon. There was no family history of colorectal cancer. The patient underwent a left hemicolectomy with regional lymphadenectomy. The tumor measured $5.2 \times 4.5 \times 0.7 \mathrm{~cm}$ and histology showed an ulcerated, moderately differentiated, adenocarcinoma of the colon, that infiltrated the pericolic fat. Fifteen resected lymph nodes were negative for metastasis (stage T3N0 according to the American Joint Committee on Cancer Seventh Edition). There were no signs of inflammation that could suggest the existence of indolent ulcerative colitis in the colectomy specimen. No testing for hallmarks of Lynch syndrome was performed (microsatellite instability/mismatch repair defect). Subsequently, the patient received 12 cycles of adjuvant chemotherapy with oxaliplatin/leucovorin/5-fluorouracil (FOLFOX6), without any complications. Thereafter, the patient was in a frequent surveillance program, without any significant findings either in laboratory or imaging studies. Notable to mention is that all follow-up colonoscopies performed did not show any pathology.

Six years after the occurrence of colon cancer, the patient was admitted again in our hospital due to hematochezia. Blood tests and imaging studies were all normal. Fecal cultures and testing for parasites were negative. Endoscopic evaluation with colonoscopy revealed pathology in the entire length of the large intestine (pancolitis), with the bowel mucosa appearing red, edematous, and friable, with a microgranular texture.

Histopathologically, in sections of the large bowel mucosa, a hemorrhagic tendency was found in the lamina propria as well as significant infiltration with lymphocytes, eosinophils, and polymorphonuclear cells, with accompanying cryptitis (Figure 1). Inflammation extended to the submucosal layer. The amount of mucus production was normal but the architecture of the small glands was slightly disrupted (unification of crypts, Figure 2). The original specimen of the colorectal cancer resection was reviewed again by the pathologist and no signs of ulcerative or any other type of colitis were found. Computed tomography scan of the abdomen did not show any pathologic findings.

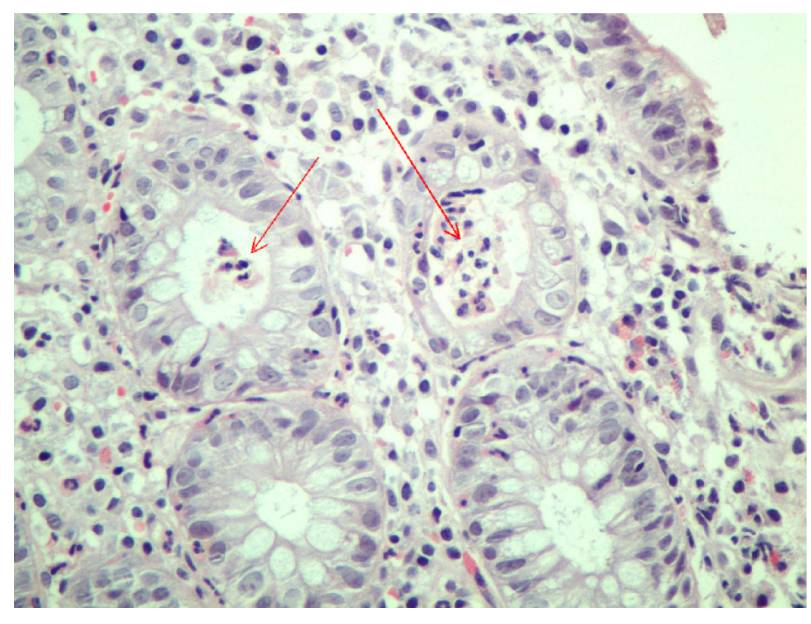

Figure I Mixed type inflammatory cells in the lamina propria, distortion of crypt architecture, and frank crypt abscesses (arrows).

Based on the above findings, a diagnosis of ulcerative colitis was made, and the patient was started on an oral regimen of mesalazine. The patient's symptoms improved significantly and after a few days he was discharged from the hospital. After one month, the patient remained symptom-free and returned back to his normal follow-up program.

\section{Discussion}

To our knowledge, there are not many reported cases in the literature in which CRC precedes ulcerative colitis by so long. Cases like this are quite rare, which could be due to the fact that CRC is mainly a disease of older age, with $90 \%$ of cases occurring after the age of 50 years whereas most cases of ulcerative colitis occur between the ages of 15 and 40 years. However, some studies suggest a bimodal age distribution for inflammatory bowel disease, with a second peak between the ages of 50 and 80 years. ${ }^{8}$ Ulcerative colitis

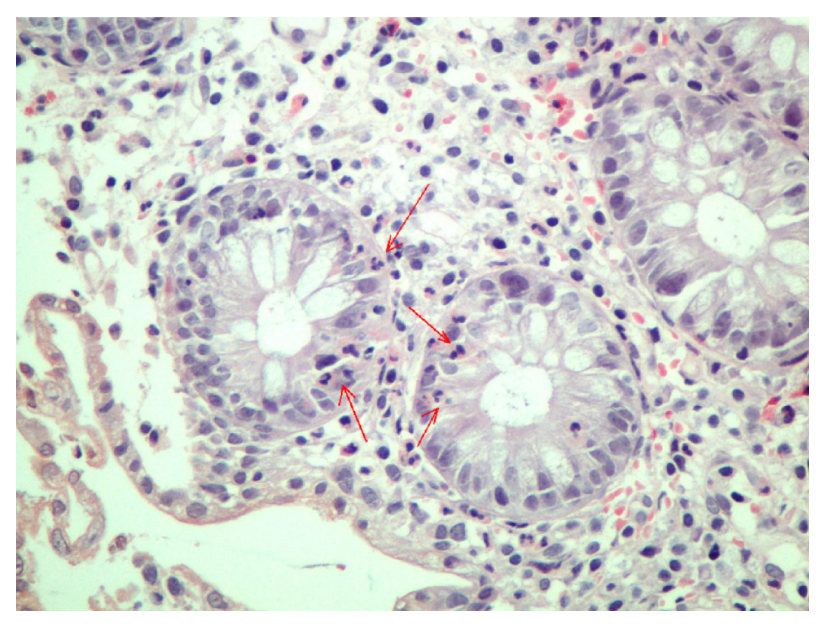

Figure 2 Polymorphonuclear infiltration of crypt mucosal cells (arrows). 
and $\mathrm{CRC}$ are two separate entities, and theoretically the two diseases could coexist in the same patient independently. So, one can assume that in our case (and every time CRC precedes ulcerative colitis), the two entities are unrelated. But is this always the case?

Patients with ulcerative colitis have a greater risk for development of CRC. It is thought that chronic inflammation is the factor playing the most decisive role. ${ }^{910}$ However, things seem to be more complicated because it is difficult to estimate the exact effect of inflammation. ${ }^{11}$ Sometimes it is even difficult to detect inflammation as the etiologic factor. It is known that malignancy can occur without the presence of microscopic inflammatory findings and without low-grade dysplasia that evolves to high-grade dysplasia first. ${ }^{12,13}$ Additionally, it was found from patients with ulcerative colitis who underwent colectomy that some of the patients with mild disease on endoscopy were found to have worse pathologic findings in the excised specimen. ${ }^{11,14}$ Additionally, in left-side colitis, it is possible that cancer occurs in areas seemingly normal macroscopically, centrally to the visible inflammatory lesions. Despite the fact that the endoscopic picture we get with colonoscopy satisfactorily reflects the extent and degree of inflammation, discrepancies can occur between endoscopic findings and histopathologic findings. ${ }^{11,13}$ Even if the endoscopic study suggests remission of the disease and the mucosa appears normal, microscopic abnormalities can persist in histopathology. ${ }^{13}$ Furthermore, many patients who develop CRC do so on a background of disease that is in a quiescent stage both histologically and clinically. ${ }^{11,14}$

Therefore, it is possible that our patient had quiescent ulcerative colitis that had remained asymptomatic and undiagnosed, then developed CRC, and had exacerbation of his colitis 6 years later. There are known cases where ulcerative colitis was incidentally diagnosed in specimens obtained from bowel segments during surgical treatment of CRC. ${ }^{7}$

Ulcerative colitis can sometimes cause only subtle symptoms that can go unnoticed by the patient, or they can be attributed to benign conditions, such as irritable bowel syndrome. The degree of inflammation can be so mild that the patient might not have any symptoms at all for years. However, in the case of our patient, colonoscopies revealed no suspicious findings and normal tissue specimens obtained from the tumor surroundings showed no signs of pathology typical for ulcerative colitis. ${ }^{7}$ This means that, although the pre-existing dysplastic lesion from which the tumor emerged could not be identified, at the time of diagnosis of CRC the patient most probably had no ulcerative colitis.
Other possibilities to explain the late occurrence of ulcerative colitis in our case could be the occurrence of a late effect of chemotherapy, or the existence of underlying common factors that make the bowel susceptible to both ulcerative colitis and CRC. Patients undergoing chemotherapy are known to experience various adverse effects. Some of them can be severe and life-threatening, such as hematologic or cardiovascular toxicities, while others are mild. Gastrointestinal side effects are very common and sometimes can be severe enough to require medical intervention. All three agents that our patient received (5-fluorouracil, leucovorin, oxaliplatin) are known to cause colitis-like symptoms, but it is highly unlikely that they could have been the cause of ulcerative colitis 6 years later. Up to $49 \%$ of long-term CRC survivors report chronic diarrhea, but this is not related to occurrence of inflammatory bowel disease like ulcerative colitis. ${ }^{15}$

The association between ulcerative colitis and CRC has been known for decades, but questions remain as to whether ulcerative colitis is the culprit in development of CRC or if the two entities simply share common pathogenetic mechanisms that remain unknown. Genetic factors play an important role in both ulcerative colitis and CRC. It is now known that in ulcerative colitis there are abnormalities found in normal epithelial cells adjacent to the dysplastic cells, and even in the entire colon. These abnormalities refer to aneuploidy, p53 mutations, and clonal chromosomal defects. ${ }^{12}$

However, despite these advances, further research is required in the direction of identifying common molecular pathways. In the case of ulcerative colitis, these genetic factors trigger a pathologic immune response. There has been a longstanding debate as to whether even sporadic CRC is strongly associated with a state of continuous microinflammation in the colon, triggered by bacteria of the intestinal flora or with a disorder in the normal regulatory response of the immune system towards these bacteria. ${ }^{10}$ This is supported by the fact that the differences between sporadic CRC and inflammatory bowel disease-related CRC are by far outweighed by their similarities, that include sequence from low-grade dysplasia to cancer, similar frequency of main chromosomal abnormalities, similar glucosylation changes, and above all, a similar prognosis. ${ }^{6,9}$

\section{Conclusion}

Ulcerative colitis and CRC are two closely correlated entities, but the exact nature of this correlation remains largely unknown. Much effort must be made to clear up this correlation in order to optimize our clinical practice and surveillance guidelines. 


\section{Disclosure}

The authors report no conflicts of interest in this work.

\section{References}

1. Danese S, Fiocchi C. Ulcerative colitis. $N$ Engl J Med. 2011;365: 1713-1725.

2. Langan RC, Gotsch PB, Krafczyk MA, Skillinge DD. Ulcerative colitis: diagnosis and treatment. Am Fam Physician. 2007;76:1323-1330.

3. Siegel R, Naishadham D, Jemal A. Cancer statistics, 2013. CA Cancer J Clin. 2013;63:11-30.

4. Watson AJM, Collins PD. Colon cancer: a civilization disorder. Dig Dis. 2011;29:222-228.

5. Ahmadi A, Polyak S, Draganov P. Colorectal cancer surveillance in inflammatory bowel disease. The search continues. World $J$ Gastroenterol. 2009;15:61-66.

6. Breynaert C, Vermeire S, Rutgeerts P, Van Assche G. Dysplasia and colorectal cancer in inflammatory bowel disease: A result of inflammation or an intrinsic risk? Acta Gastroenterol Belg. 2008;71:367-372.

7. Katsanos KH, Christodoulou D, Siozopoulou V, et al. Silent ulcerative colitis adjacent to a regular sigmoid adenocarcinoma. Eur J Gastroenterol Hepatol. 2011;23:957-960.

8. Ekbom A, Helmick C, Zack M, Adami HO. The epidemiology of inflammatory bowel disease: a large, population-based study in Sweden. Gastroenterology. 1991;100:350-358.
9. Triantaffilidis J, Nasioulas G, Kosmidis P. Colorectal cancer and inflammatory bowel disease: epidemiology, risk factors, mechanisms of carcinogenesis and prevention strategies. Anticancer Res. 2009;29: 2727-2737.

10. Rhodes JM, Campbell BJ. Inflammation and colorectal cancer: IBDassociated and sporadic cancer compared. Trends Mol Med. 2002;8: 10-16.

11. Itzkowitz SH, Yio X. Inflammation and cancer IV. Colorectal cancer in inflammatory bowel disease: the role of inflammation. Am J Physiol Gastrointest Liver Physiol. 2004;287:G7-G17.

12. Risques RA, Lai LA, Himmetoglu C, et al. Ulcerative colitis-associated cancer arises in a field of short telomeres, senescence and inflammation. Cancer Res. 2011;71:1669-1679.

13. Ando T, Nishio Y, Watanabe O, et al. Value of colonoscopy for prediction of prognosis in patients with ulcerative colitis. World $J$ Gastroenterol. 2008;14:2133-2138.

14. Riley SA, Mani V, Goodman MJ, Dutt S, Herd ME. Microscopic activity in ulcerative colitis: what does it mean? Gut. 1991;32: 174-178.

15. Ramsey SD, Berry K, Moinpour C, Giedzinska A, Andersen MR. Quality of life in long term survivors of colorectal cancer. $\mathrm{Am} \mathrm{J}$ Gastroenterol. 2002;97:1228-1234.
International Medical Case Reports Journal

\section{Publish your work in this journal}

The International Medical Case Reports Journal is an international, peer-reviewed open-access journal publishing original case reports from all medical specialties. Previously unpublished medical posters are also accepted relating to any area of clinical or preclinical science. Submissions should not normally exceed 2,000 words or

\section{Dovepress}

4 published pages including figures, diagrams and references. The manuscript management system is completely online and includes a very quick and fair peer-review system, which is all easy to use. Visit http://www.dovepress.com/testimonials.php to read real quotes from published authors. 